\title{
Novel Solutions for Smart Cities - Creating Air Pollution Maps Based on Intelligent Sensors
}

\author{
Marzena Banach \\ Institute of Architecture and Spatial Planning, \\ Poznań University of Technology, \\ Nieszawska 13C, 61-021 Poznań, Poland, \\ E-mail: marzena.banach@erba.com.pl
}

\author{
Tomasz Talaśka and Rafał Długosz \\ UTP University of Science and Technology, \\ Faculty of Telecommunication, Computer Science and \\ Electrical Engineering, \\ ul. Kaliskiego 7, 85-796, Bydgoszcz, Poland, \\ E-mail: talaska@utp.edu.pl,rafal.dlugosz@gmail.com
}

\begin{abstract}
The paper presents novel solutions for systems used to create air pollutions maps in smart cities. Ability to record pollution levels with a function of a short-term prediction of their fluctuations may be useful for cyclists and pedestrians moving through the city. Based on such data they can choose their route through the city in such a way, as to avoid the most polluted areas. Systems of this type are in the range of solutions characteristic for smart cities. Their effectiveness requires a relatively dense wireless sensor network (WSN) composed of miniaturized and cheap intelligent pollution sensors, capable not only of data recording and transmitting, but also of some data processing with the prediction abilities. Sensors of this type require a development of various circuit components that feature small sizes and ultra-low energy consumption. One of the main blocks, in this case, should be an artificial neural network (ANN) implemented at the transistor level. In this work, we present prototype circuits designed by us for the described purposes. The realized blocks include a finite impulse response (FIR) filter, programmable analog-to-digital converters (ADCs) with internal controlling clock generators and main building blocks of a parallel ANN. The specialized chips (ASIC - application specific integrated circuit) with the described components were implemented in the CMOS technology in the full custom style.
\end{abstract}

\section{INTRODUCTION}

C ITIES are not homogeneous systems. Depending on the area, different may be the terrain as well as urban occupation. The type of buildings includes such parameters as its density and height, width, and location of streets in relation to wind directions, distribution of green areas, etc.

Particular areas of the cities play a different function, which often translates into the intensity of traffic, which is one of the main factors causing pollutions. The described factors affect the natural possibilities of ventilation and the absorption of pollutants, and thus the susceptibility of particular areas of the city to changes in pollution levels. An important factor here is the time of persistence of specific levels of pollutions, even after the expiry of stimuli affecting them.

Air pollution is also affected by the seasons. This is due not only to the fact of a larger emission due to heating but also to natural changes in green areas. The biologically active surface (lawns, parks, green walls, green roofs, trees) has a significant impact on the absorption of pollutants.

In such cities as Kraków in Poland, the terrain does not support the natural ventilation, so the pollution levels are frequently at high levels. On the other hand, when designing new cities, this factor may be taken into account. An example here is the Zenata Eco-city developed in Morocco near the city of Casablanca. One of the examples of the smart approach at the city's design stage is taking into account natural conditions. For example, the wind directions in the area were examined in order to build the city in such a way, to allow for natural ventilation and lowering the temperature by several degrees in the summer [18]. It is also planned to collect rainwater in retention reservoirs and use it to maintain green areas - in plans, $30 \%$ of the area of the city will be covered by greenery (compared to $3 \%$ in Casablanca).

With such a diversity of conditions, relying only on a few or a dozen stations measuring levels of pollutions in a given area is not sufficient. At present, in main cities in Poland, on average, there are only a few to a dozen or so automatic stations measuring the pollution levels [1], [2].

The price of currently offered devices and their sizes do not support dense maps. To solve this problem, it is necessary to significantly reduce the price of a single device. The solution here may be miniaturized integrated sensors realized in the CMOS technology, implemented either as a System-on-Chip or as a System-in-Package. As shown in the next section, examples of the implementation of integrated pollution sensors can be already found in the literature. This is an important step towards advanced small and cheap measuring devices.

Another need is to increase the prediction abilities offered by the monitoring systems, especially for the short time horizon. Such information may be of fundamental importance, for example for cyclists and pedestrians moving around the city. Based on such data, they may consciously choose particular sections of their route through the city. The next step in the development of the monitoring systems may be the implementation of more complex measurement devices that offer an onboard ability to make predictions, fully independently from the main station.

The paper has the following structure. In the next Section, a state-of-the-art study is presented. Pollution monitoring systems cover many different elements that need to be presented separately. This part first discusses the monitoring systems considered as a whole. Subsequently, details relevant to the implementation of the integrated measuring devices are described. Then examples of the use of ANNs in the pollution 
prediction problem are being presented. In the following Section, the authors' contribution to the development of integrated intelligent sensors is described, with such circuit components of such devices, as Analog-to-Digital Converters (ADCs), filters and components of the ANN. In the last Section, the conclusions are drawn.

\section{STATE-OF-THE ART STUDY}

\section{A. Air pollution monitoring systems}

In Poland, Voivodship Environmental Protection Inspectorates provide data from environmental monitoring for particular voivodships [3]. The offered system is based mainly on networks of measurement stations located in sensitive points of voivodships - mainly in large cities. Such stations measure concentrations of, among others, such gases and substances as sulfur dioxide, nitrogen oxides, benzene, carbon monoxide, ozone, suspended dust $P M_{10}$ and $P M_{2.5}$. In 2017, in Poland, air quality measurements were carried out by 1,924 measuring stations, including 1,098 automated stations (57\%).

Airly company develops its own system, which is supposed to be much denser. Currently, the highest density of measuring devices is offered in Kraków. The measurement results offered to the public throughout the company's webpage show, this is justified, as concentrations can change significantly in short periods of time, while large differences may be visible even between areas located close to each other. Selected results from the Airly website, are shown in Figure 1. Diagrams (a) and (b), show results for two following hours (one Saturday in May) for the overall city.

At this point, it is worth mentioning wearable pollution sensors already offered on the market. Their usage is limited, as they require a person equipped with such a sensor to be present in a given area of the city. It does not protect this person from the effects of the pollutions. A better solution would be stationary sensors providing data to a central computation station, which would be able to perform a shortterm prediction based on the collected data. In this way, city users could plan their route or the mean of transport more consciously in advance. One of the useful options related to the described wearable sensors would be sharing collected data with a central system so that other people could use. Such an approach could support the stationary system, increasing the prediction abilities and the map resolution.

\section{B. Microelectronic sensors of pollution particles}

In the literature, one can find several examples of the implementation of particle sensors of various air contaminations. Texas Instruments company proposed an optoelectronic system for the detection and measurement of the $\mathrm{PM}_{2.5}$ and the $\mathrm{PM}_{10}$ particles. The system is based on the detection of scattered light by particles suspended in the air [14]. In [15] presented are methods of implementing the $\mathrm{PM}_{2.5}$ and $\mathrm{PM}_{10}$ particle micrometer using the zinc oxide based on the Solidly Mounted Resonator (SMD). The authors of [15] present a complete system mounted on a printed board (PCB). On the board, apart from the particle sensor $\mathrm{PM}_{10}$ and $\mathrm{PM}_{2.5}$, there is also
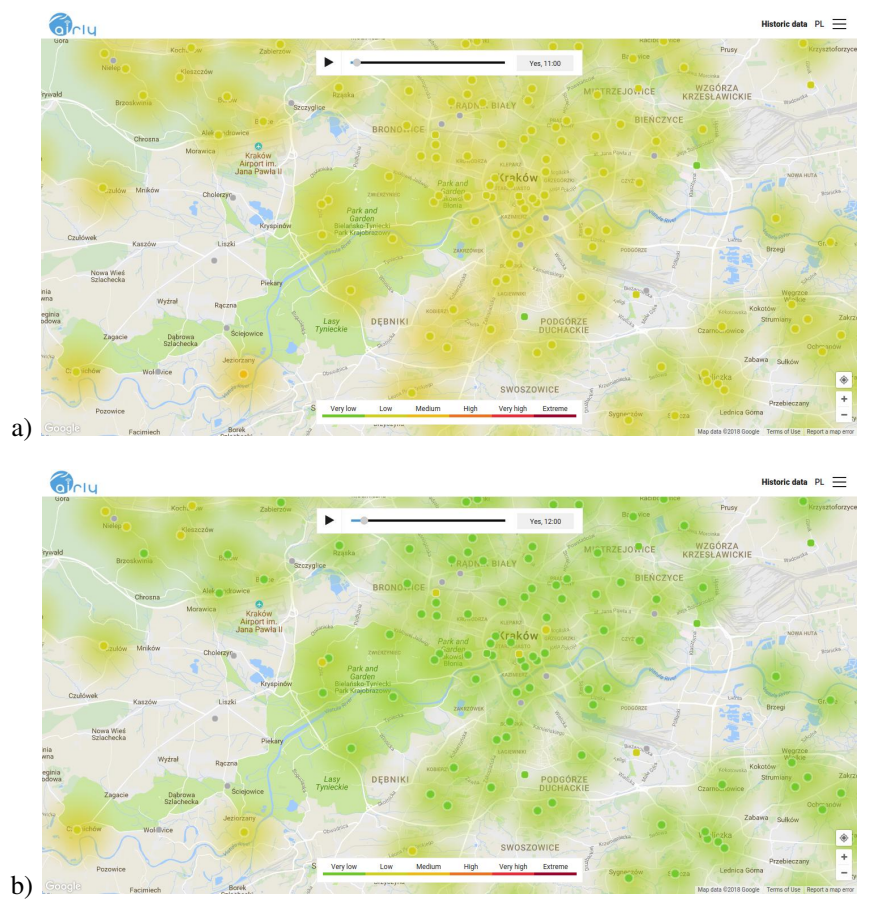

Fig. 1. Selected maps obtained from the Airly company's webpage (source: Airly sp. z o.o., Aleja Pokoju 1a, 31-548, Kraków, Poland, https://map.airly. $\mathrm{eu} / \mathrm{pl} /$ ) for the overall city for two following hours.

a specialized ASIC, used by the sensor. The system works correctly, however, it consists of two separate blocks - the sensor and the system managing it. This is a disadvantage, for it increases the dimensions of the device, and what is also important, in such a solution there is a lack of encapsulation of the electronic circuits, which is important taking into account sometimes hard environmental conditions.

Another example is an integrated capacitive sensor designed in the CMOS $0.35 \mu \mathrm{m}$ technology [16], [17]. The advantage of this system is the fact that it is implemented inside the integrated circuit, which enables, apart from the detection of molecules, also other parallel operations. The system is capable of detecting microscopic particle sizes of pollutants. This work is an important novelty in this area in the world, as the sensor solutions inside the integrated circuit are presented for the first time here.

The availability of the described works is very important. The combination of such solutions with the ANN offered by the authors of this article is important from the point of view of the development of fully intelligent and autonomous sensors.

\section{Using ANN to monitor and predict air pollutants}

Development of smart cities would not be possible without the use of artificial intelligence (AI) algorithms, including artificial neural networks (ANN), genetic algorithms (AG), fuzzy systems (FS), or expert systems (SE). AI will play a significant role in the development and functioning of such cities. On one hand, this is due to the very large complexity of the system which the city is, and on the other hand, the 
lack of the possibility of an accurate mathematical description of the interdependencies between various parameters of this system. Complexity means also a huge amount of data, which is often difficult to express unequivocally with the help of unified indicators.

ANNs are universal tools frequently used in different areas of daily life. They are frequently employed for data classification, prediction, recognition, detection, etc. Neural networks operate more effectively if they receive properly prepared data (e.g. normalized) and when they have the sufficiently large computing power to process them within an acceptable time interval. It is also necessary to match a given type of the ANN to a specific problem. One of the areas in which these tools are more and more frequently used is the problem of air pollution, described in this paper. In this case, they are capable of contamination forecasting [11], [12], [6], [5], [13], [10], [7], [4].

One of the examples here, is the application of the MultiLayer Perceptron (MLP) and the Radial Basis Function (RBF) neural networks for a long-term prediction of pollutants dust $\left(\mathrm{PM}_{10}, \mathrm{PM}_{2.5}\right)$ (especially in cities) [11], [12]. In another work [6] a multilayer perceptron (MLP) has been used to forecast the impact of pollution caused by road traffic (exhaust fumes) on the health of residents. In [13] three different machine learning algorithms are presented, including, among others, a neural network for the monitoring and forecasting of pollutants, such as ground-level ozone $\mathrm{O}_{3}$, nitrogen dioxide $\mathrm{NO}_{2}$ and dioxide sulfur $\mathrm{SO}_{2}$. Another work in this area [10] presents the concept of self-organizing networks [7] used for the classification of data on sulfur dioxide hazards $\mathrm{SO}_{2}$ is presented.

\section{PRoposed CONTRIBUtion to THE DEVELOPMENT OF INTELLIGENT POLLUTION SENSORS}

In this Section, we present a model of an integrated system for the detection and prediction of air pollution particles in the form of a specialized chip implemented in the CMOS technology. State-of-the art works in this area, described above, focus on the implementation of either the pollution particles sensors in the form of an integrated circuit or the use of software implementation of neural networks to detect impurities. In this work, we present the solutions that will allow joining those two areas into a single integrated device, capable of working at WSN.

The proposed intelligent sensor consists of several main components, such as: (i) air pollution sensor (a standard solution) used to collect data from the city, (ii) a filter used to remove noises from the signal, (iii) an ADC used to convert measured analog data into a digital signal further processed in, (iv) data processing unit with a hardware realized ANN. The processed data are transferred to the base station using (v) an RF communication block (standard block to be used).

The neural network, that can be used in the sensor, may be either analog or digital. In the second case, a preliminary analog-to-digital conversion with the appropriate resolution is required. Even though, the preferred solution is the use of a

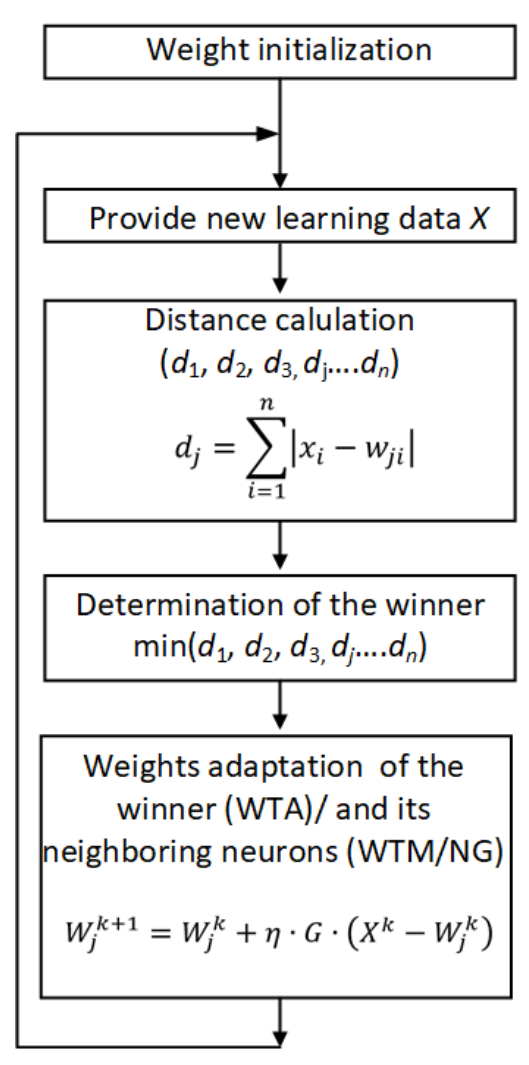

Fig. 2. Self-organizing neural network algorithm.

digital neural network, due to its robustness to various types of external conditions and the impact of the imperfections of the technological process. Both filters and ADCs were previously designed by us [19]. A prototype ASIC containing these components is shown in Figure 3.

In this work, we focus on ANN, that will be integrated directly with other components of the sensor. In the proposed approach we use self-organizing networks since such networks are already used with success for the analysis and prediction of the air pollutions. Moreover, such networks offer a relatively simple structure, which is crucial from the point of view of the implementation in the ASIC. Low mathematical complexity allows for a large miniaturization. The structure of such a network is schematically shown in Figure 2. The basic operations performed for each learning pattern $X$ are as follows:

- initialization of the neuron weights,

- providing new learning pattern $X$ from the ADC and indirectly from the pollution sensor,

- calculation of distances $\left(d_{1}, d_{2}, d_{3}, d_{j}, \ldots, d_{n}\right)$ between the learning pattern $X$ and weight vectors $W$ of particular neurons. One of the typical distance measures may be used, namely Manhattan or Euclidean one [8],

- determination of the winning neuron. Mathematically it is the $\min \left(d_{1}, d_{2}, d_{3}, d_{j}, \ldots, d_{n}\right)$ operation,

- determination of the neighborhood of the winning neuron 


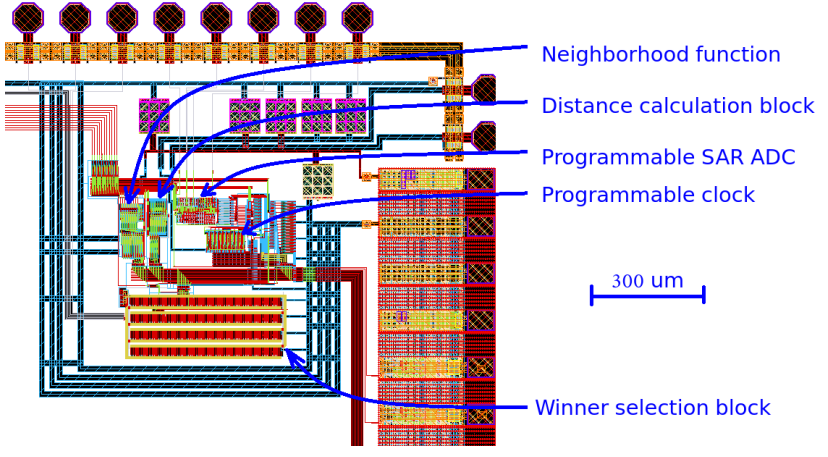

Fig. 3. A prototype ASIC designed by us for the application in the intelligent sensors, containing components of the ANN and a programmable SAR ADC, controlled by internal programmable clock generator.

and the calculation of the learning rate $\eta$ for particular neighbors,

- adaptation of the winning neuron (in WTA only this neuron is adapted) and its neighbors (in WTM and NG). The adaptation is realized according to a formula:

$$
W_{j}(k+1)=W_{k}(l)+\eta(k) \cdot G() \cdot\left[X(k)-W_{j}(k)\right]
$$

In this formula $W_{k}$ is the weights vector of a $j^{\text {th }}$ neuron. The neurons that belong to the winner's neighborhood, are trained with the intensities determined by the applied neighborhood function $G()$ [8], [9].

The prototype chip, shown in Fig. 3, contains most of the components of the proposed intelligent sensor, including particular building blocks of the self-organizing neural network described earlier. Particular subcircuits of this chip are responsible for the computations performed at particular stages of the learning algorithm of the $\mathrm{NN}$, as described above. The chip also contains an ultra-low power, programmable, 10bits ADC. All these components were tested by means of laboratory measurements.

\section{CONCLUSION}

The paper presents the concept of a system for the monitoring of air pollution in smart cities. The proposed concept is based on the usage of a dense network of miniature intelligent pollution sensors. The assumption is to build cheap sensors, self-sufficient in energy so that they can be easily deployed on city streets without having access to the power line.

The implementation of such sensors requires the development of particular building components. A special attention should be paid to the sizes of these blocks and their computing power while maintaining their functionality in the comparison with similar typical software solutions.

For this reason, we presented selected hardware solutions designed by us, with particular emphasis on miniature artificial neural networks capable of working in parallel.

In this work, we present intermediate results. The next step will be to assemble the designed blocks into a larger system.
It is worth to add, that the proposed solutions can cooperate not only with the pollution sensors. Their usage is universal. A modular structure of the resulting device may be considered, in which the sensor would be an element selected depending on the needs, while the signal processing scheme would be similar.

\section{REFERENCES}

[1] https://map.airly.eu/pl/, (access 2018.09.10).

[2] http://aqicn.org/map/world/, (access 2018.09.10).

[3] http://sojp.wios.warszawa.pl/, (access 2018.09.10).

[4] Moustris K. P., Larissi I. K., Nastos P. T., Koukouletsos K. V., Paliatsos A. G., "Development and Application of Artificial Neural Network Modeling in Forecasting PM10 Levels in a Mediterranean City," Water Air Soil Pollut, DOI 10.1007/s11270-013-1634-x, 224:1634, 2013

[5] Sahina U.A., Bayatb C., Uçan O.N., "Application of cellular neural network $(\mathrm{CNN})$ to the prediction of missing air pollutant data," Atmospheric Research, Elsevier, Vol.101, s.314-326, 2011.

[6] Fontes T., Silva L.M., Pereira S.R., Coelho M.C., "Application of Artificial Neural Networks to Predict the Impact of Traffic Emissions on Human Health. Progress in Artificial Intelligence," Springer, Berlin, Heidelberg, Lecture Notes in Computer Science, Vol.8154, s.21-29, 2013.

[7] Kohonen T., "Self-Organizing Maps (Information Sciences)," trzecie wydanie, Nowy Jork (USA), Springer-Verlag, 2001.

[8] Długosz R., Kolasa M., Pedrycz W., Szulc M., "Parallel programmable asynchronous neighborhood mechanism for Kohonen SOM implemented in CMOS technology," IEEE Transactions Neural Networks, Vol. 22, No. 12, s.2091-2104, December 2011.

[9] Talaśka T., Długosz R., "Analog, parallel, sorting circuit for the application in Neural Gas learning algorithm implemented in the CMOS technology," Applied Mathematics and Computation, Vol. 319, 2018

[10] J. M. Barrón-Adame and O. G. Ibarra-Manzano and A. Vega-Corona and M. G. Cortina-Januchs and D. Andina, "Air pollution data classification by SOM Neural Network," World Automation Congress 2012, pp.1-5, 2012.

[11] W. Kaminski and J. Skrzypski and E. Jach-Szakiel, "Application of Artificial Neural Networks (ANNs) to Predict Air Quality Classes in Big Cities," International Conference on Systems Engineering, pp.135140, 2008.

[12] M. M. Dedovic and S. Avdakovic and I. Turkovic and N. Dautbasic and T. Konjic, "Forecasting PM10 concentrations using neural networks and system for improving air quality," International Symposium on Telecommunications (BIHTEL), pp.1-6, 2016.

[13] K. Bashir Shaban and A. Kadri and E. Rezk, "Urban Air Pollution Monitoring System With Forecasting Models," IEEE Sensors Journal, vol. 16, no. 8, pp.2598-2606, 2016.

[14] "PM2.5 and PM10 Particle Sensor Analog Front-End for Air Quality Monitoring Reference Design," Texas Instruments Incorporated, http://www.ti.com/tool/TIDA-00378

[15] S. Thomas and F. H. Villa-López and J. Theunis and J. Peters and M. Cole and J. W. Gardner, "Particle Sensor Using Solidly Mounted Resonators,' IEEE Sensors Journal, vol. 16, no. 8, pp.2282-2289, 2016.

[16] P. Ciccarella and M. Carminati and M. Sampietro and G. Ferrari, "Multichannel 65 zF rms Resolution CMOS Monolithic Capacitive Sensor for Counting Single Micrometer-Sized Airborne Particles on Chip," IEEE Journal of Solid-State Circuits, vol. 51, no. 11, pp.25452553, 2016.

[17] M. Carminati and P. Ciccarella and M. Sampietro and G. Ferrari, "Single-Chip CMOS Capacitive Sensor for Ubiquitous Dust Detection and Granulometry with Sub-micrometric Resolution," Springer International Publishing AG 2018, Sensors, Lecture Notes in Electrical Engineering, pp.8-18, 2018.

[18] Christin Roby, "An inside look into Africa's first eco-city: Zenata, Morocco," devex, 13 March 2017, https://www.devex.com/news/ an-inside-look-into-africa-s-first-eco-city-zenata-morocco-89741

[19] Długosz R. and Fischer G. "Low chip area, low power dissipation, programmable, current mode, 10-bits, SAR ADC implemented in the CMOS 130nm technology," International Conference Mixed Design of Integrated Circuits and Systems (MIXDES), Poland, 2015. 